\title{
Lowering iodinated contrast concentration in infrainguinal endovascular interventions: a three-armed randomized controlled non-inferiority trial
}

\author{
Sjoerd Jens ${ }^{1}$ - Sanne M. Schreuder ${ }^{1}$ - Diederik W. De Boo ${ }^{1} \cdot$ Lukas C. van Dijk $^{2}$ • \\ Hans van Overhagen ${ }^{2} \cdot$ Shandra Bipat $^{1} \cdot$ Mark J. W. Koelemay $^{1} \cdot$ Jim A. Reekers ${ }^{1}$
}

Received: 2 June 2015 /Revised: 3 November 2015 / Accepted: 10 November 2015 /Published online: 2 December 2015

(C) The Author(s) 2015. This article is published with open access at Springerlink.com

\begin{abstract}
Objectives To determine the influence of lowering iodinated contrast concentration on confidence of interventional radiologists in diagnosing and treating lesions during endovascular interventions in patients with symptomatic peripheral arterial disease (PAD).

Methods A randomized controlled non-inferiority trial was performed in 60 patients. Intervention was performed with contrast concentrations (in $\mathrm{mg}$ of iodine per $\mathrm{mL}, \mathrm{mgI} / \mathrm{mL}$ ) of 300 (standard), 240 , or $140 \mathrm{mgI} / \mathrm{mL}$. Primary outcome was confidence (score $0-100 \%$ ) of radiologists in diagnosing and treating arterial lesions. Secondary outcomes were procedural iodine load and image quality (i.e. non-diagnostic, limited, diagnostic, exemplary).

Results Median confidence scores in diagnosing lesions were $100 \%$ (range $81-100 \%$ ) for the 300 group ( $=21), 100 \%$ (range $82-100 \%$ ) for the 240 group ( $\mathrm{n}=19$ ), and $100 \%$ (range 91-100 \%) for the 140 group ( $\mathrm{n}=20$ ) (both $p=1.00$ compared to the 300 group). Median scores for treating lesions in the 240 and 140 groups, $100 \%$ (range $79-100 \%, p=0.40$ ), and $100 \%$ (range 63-100\%, $p=0.25$ ), respectively, were not lower compared to the 300 group (median $100 \%$, range 78-100\%). Procedural iodine load was lower in the $240(24.3 \pm 7.6 \mathrm{~g}$, $p=0.022)$ and 140 groups $(17.8 \pm 5.6 \mathrm{~g}, p<0.001)$ compared
\end{abstract}

Presentation of study This randomized controlled trial has been presented at CIRSE in September 2015.

Sjoerd Jens

s.jens@amc.uva.nl

1 Department of Radiology, Academic Medical Center, Room G1-229, Meibergdreef 9, 1105 AZ Amsterdam, The Netherlands

2 HagaZiekenhuis, Leyweg 275, 2545 CH Den Haag, Netherlands to the 300 group $(29.7 \pm 6.3 \mathrm{~g})$. Image quality was diagnostic for all groups.

Conclusion Using iodine contrast of $140 \mathrm{mgI} / \mathrm{mL}$ for diagnosis and interventions in PAD patients significantly reduces administered iodine load without compromising image quality. Future use of lower iodine dose is recommended.

Key Points

- Lower iodinated contrast concentration during endovascular intervention does not decrease radiologist's confidence.

- Image quality of standardized angiographies remains diagnostic using $140 \mathrm{mgI} / \mathrm{mL}$ iodinated contrast concentration.

- Iodine load during intervention can be decreased by $>40 \%$ when using $140 \mathrm{mgI} / \mathrm{mL}$.

- Implementing the use of a lower iodinated contrast concentration will reduce the costs of the procedure.

Keywords Peripheral arterial disease $\cdot$ Intermittent claudication · Critical limb ischemia Contrast media . Angiography

\section{Introduction}

The use of intravascular iodinated contrast media carries a risk of contrast-induced nephropathy (CIN), which is associated with an increase in morbidity and mortality [1]. CIN can be defined as an impairment in renal function, i.e. an increase in serum creatinine by more than $25 \%$ or $44 \mu \mathrm{mol} / \mathrm{L}$, occurring within 3 days following intravascular contrast administration without an alternative aetiology [2]. The incidence of CIN is low in patients with a normal renal function, but can be as high as $25 \%$ in patients with impaired renal function, especially secondary to diabetes mellitus [3]. 
Patients with intermittent claudication (IC) or critical limb ischemia (CLI) due to peripheral arterial disease (PAD), often have several risk factors for developing CIN after an endovascular intervention. To prevent $\mathrm{CIN}$ in high risk patients, hydration using saline or bicarbonate is recommended, but based on the consensus of experts and small or retrospective studies [1]. Other strategies, such as $N$-acetylcysteine [4] or statin pretreatment [5], are also accepted approaches to prevention of CIN, though not evidence-based.

In the 1980s and 1990s, many studies were performed on finding the right contrast medium and concentration [6, 7]. In 1992, Ugolotti et al showed that with concentrations of $150 \mathrm{mg}$ iodine per $\mathrm{mL}(\mathrm{mgI} / \mathrm{mL})$ adequate images of the lower limb could be acquired [8]. With improved technical specifications for angiographic equipment achieved during the last few decades, such as a higher image resolution, lowering the iodinated contrast concentration might be an appropriate way to prevent CIN, especially since a higher volume use of iodinated contrast is associated with the risk of CIN [9]. Currently, many interventional radiologists use a contrast concentration of about $300 \mathrm{mg}$ iodine per $\mathrm{mL}(\mathrm{mgI} / \mathrm{mL})$ for lower limb angiography and interventions. Lowering this concentration might compromise the image quality, especially in patients with CLI and multi-level disease.

The aim of this study was to compare the confidence of interventional radiologists in performing an endovascular intervention in patients with PAD between three different concentrations of iodinated contrast.

\section{Materials and methods}

This trial was reported according to the extended Consolidated Standards of Reporting Trials (CONSORT) 2010 statement [10]. This trial was registered in advance at www.trialregister.nl (trial identification NTR3406). Ethical approval was obtained from the local ethics committee.

\section{Trial design}

The trial was designed as a prospective, multicentre, threearmed, double-blind randomized controlled non-inferiority trial and was carried out in two hospitals.

For the three arms, iohexol $300 \mathrm{mgI} / \mathrm{mL}$, considered the control group, iohexol $240 \mathrm{mgI} / \mathrm{mL}$, and iohexol $140 \mathrm{mgI} /$ $\mathrm{mL}$ contrast concentrations were used.

\section{Participants}

All patients ( $\geq 18$ years) presenting at the vascular surgery outpatient clinic referred for digital subtraction angiography (DSA) were eligible for this study. In all patients, vascular status was evaluated by duplex ultrasound before they were referred for intervention.

Inclusion criteria were:

- $\quad$ IC (Fontaine stage II) or CLI (Fontaine stage III or IV) due to PAD

- Ankle-brachial pressure index $(\mathrm{ABPI})<0.90$, or drop in ABPI $>0.15$ after exercise, or toe-brachial pressure index (TBPI) $<0.70$

- Duration of complaints $>2$ weeks

- Scheduled for DSA with anterograde femoral artery puncture

- Written informed consent

Exclusion criteria were:

- renal failure; serum creatinine $>130 \mu \mathrm{mol} / \mathrm{L}$

- inability to give informed consent

- participation in another study

Baseline characteristics of the included patients were recorded using a standardized case record form and comprised patient demographics, risk factors for atherosclerosis, history of coronary artery disease, transient ischemic attack (TIA) or stroke, family history of cardiovascular disease, and renal failure.

\section{Patient characteristics}

Sixty consecutive patients were included between April 2012 and July 2014, of which 21, 19, and 20 were randomly allocated to respectively the control, 240 , and 140 groups. The mean age of all patients was $70.8 \pm 9.5$ years, $38(63 \%)$ were male, 26 (43\%) had diabetes, and 35 (58\%) had CLI (Table 1).

\section{Interventions}

Endovascular interventions were performed by interventional radiologists who had experience in interventional radiology varying between 1 and 25 years. In total, seven interventional radiologists performed procedures for this trial.

Diagnostic DSA and intervention were done with three different doses of contrast media. In the control group, iohexol 300 was used, and in the experimental groups, iohexol 240 and iohexol 140 contrast (respectively, Omnipaque 300, 240, and $140 \mathrm{mgI} / \mathrm{mL}$, Amersham Health, Buckinghamshire, UK).

After antegrade puncture of the common femoral artery, angiography was performed according to a standardized procedure. Angiography of the superficial femoral artery and popliteal artery was done through a 5 French endhole catheter. Using a contrast power injector, $10 \mathrm{~mL}$ of contrast was injected at a rate of $5 \mathrm{~mL} / \mathrm{s}$, and an anteroposterior angiography was made. The catheter tip was then placed in the 
Table 1 Baseline characteristics

\begin{tabular}{|c|c|c|c|c|}
\hline & Total & $\begin{array}{l}300 \text { group } \\
\text { (control) }\end{array}$ & 240 group & 140 group \\
\hline No. of patients & 60 & 21 & 19 & 20 \\
\hline Age, years (SD) & $70.8(9.5)$ & $73.1(7.6)$ & $70.2(12.4)$ & $68.8(8.0)$ \\
\hline Male sex $(\%)$ & $38(63)$ & $10(48)$ & $15(79)$ & $13(65)$ \\
\hline BMI, $\mathrm{kg} / \mathrm{m}^{2}(\mathrm{SD})$ & $25.9(6.5)$ & $26.4(7.2)$ & $25.1(3.7)$ & $26.2(8.0)$ \\
\hline Diabetes $(\%)$ & $26(43)$ & $9(43)$ & $9(47)$ & $8(40)$ \\
\hline Hypertension (\%) & $51(85)$ & $16(76)$ & $17(89)$ & $18(90)$ \\
\hline \multicolumn{5}{|l|}{ Hyperlipidemia (\%) } \\
\hline No & $18(30)$ & $4(19)$ & $6(32)$ & $8(40)$ \\
\hline Yes & $19(32)$ & $6(29)$ & $9(47)$ & $4(20)$ \\
\hline Unknown, statin use & $16(27)$ & $7(33)$ & $3(16)$ & $6(30)$ \\
\hline Unknown & $7(12)$ & $4(19)$ & $1(5)$ & $2(10)$ \\
\hline \multicolumn{5}{|l|}{ Smoking (\%) } \\
\hline Current & $29(48)$ & $10(48)$ & $7(37)$ & $12(60)$ \\
\hline Former & $5(8)$ & $2(9)$ & $2(11)$ & $1(5)$ \\
\hline Never & $26(43)$ & $9(43)$ & $10(53)$ & $7(35)$ \\
\hline CAD (\%) & $12(20)$ & $4(19)$ & $3(16)$ & $5(25)$ \\
\hline TIA or stroke $(\%)$ & $12(20)$ & $3(14)$ & $3(16)$ & $6(30)$ \\
\hline Family history of CVD (\%) & $30(50)$ & $10(48)$ & $9(47)$ & $11(55)$ \\
\hline \multicolumn{5}{|l|}{ Previous treatment $(\%)$} \\
\hline Ipsilateral revasc. & $16(27)$ & $4(19)$ & $8(42)$ & $4(20)$ \\
\hline \multicolumn{5}{|l|}{ Fontaine stage $(\%)$} \\
\hline Fontaine II & $25(42)$ & $9(43)$ & $8(42)$ & $8(40)$ \\
\hline Fontaine III & $11(18)$ & $2(9)$ & $2(11)$ & $7(35)$ \\
\hline Fontaine IV & $24(40)$ & $10(48)$ & $9(47)$ & $5(25)$ \\
\hline ABI, median (IQR) & $0.63(0.50-0.82)$ & $0.57(0.42-0.77)$ & $0.71(0.59-0.85)$ & $0.63(0.50-0.83)$ \\
\hline Absolute toe pressure, $\mathrm{mmHg}(\mathrm{IQR})$ & $42(16-66)$ & $40(0-75)$ & $45(13-64)$ & $52(18-68)$ \\
\hline
\end{tabular}

$A B I$, ankle brachial pressure index; $B M I$, body mass index; $C A D$, coronary artery disease; $C V D$, cardiovascular disease; $I Q R$, interquartile range; $S D$, standard deviation; TIA, transient ischemic attack

popliteal artery at the level of the knee joint. Standard projection images of the crural arteries, i.e. anteroposterior and oblique projections, and one lateral projection of the foot, were made with $10 \mathrm{~mL}$ of contrast at a rate of $3 \mathrm{~mL} / \mathrm{s}$. When femoropopliteal or crural arterial segments were not in the field of view during the first acquisition, a second, more distal, acquisition was made.

Arterial recanalisation was performed according to standard procedures, i.e. initial balloon angioplasty, and only in residual significant stenosis or dissection a stent was placed. At any time during the procedure, the interventional radiologist had the ability to change to the standard concentration (300 $\mathrm{mgI} / \mathrm{mL}$ ), if image quality was judged too poor for adequate diagnostic evaluation or intervention.

\section{Primary outcomes}

Primary outcomes were the confidence score in diagnosing arterial stenosis or occlusion and the confidence in treating arterial stenosis or occlusion. The confidence scores were assessed using a visual analogue scale (VAS) ranging between $0 \%$ confidence and $100 \%$ confidence. These outcomes were scored by the interventional radiologist performing the procedure.

\section{Secondary outcomes}

Secondary outcomes were volume of contrast used during intervention, image quality of the standardized angiographies, interobserver agreement in assessing arterial lesions, contrastto-noise ratio (CNR), and the pain score of the patient during contrast injection.

Image quality was assessed separately for the femoropopliteal, crural, and foot angiographies by three interventional radiologists (radiologist performing the procedure, SS, and DDB) according to the Radlex ${ }^{\circledR}$ image quality scoring criteria [11]: [0] non-diagnostic (unacceptable for diagnostic purposes); (1) limited (acceptable, with some technical defects 
but still adequate for diagnostic purposes); (2) diagnostic (acceptable, with no technical defects likely to impair diagnosis); (3) exemplary (good, most adequate for diagnostic purposes).

For each artery, i.e. superficial femoral artery, popliteal artery, tibioperoneal trunk, anterior tibial artery, posterior tibial artery, peroneal artery, plantar artery, and dorsal pedal artery, two radiologists (SS, DDB; respectively, 1 year and 3 years experience in interventional radiology) scored whether the artery was (1) fully patent or stenosis $<50 \%$, (2) stenosis $>50 \%$, or (3) occluded. Foot arteries were judged as "not assessable" if distal foot arteries were not visualized at all due to crural occlusions.

CNR was assessed by the following formula:

$\mathrm{CNR}=\frac{\left|\left(\overline{I V}_{\text {artery }}-\overline{I V}_{\text {tissue }}\right)\right|}{S D_{\text {tissue }}}$

Here, the mean intensity value in the artery $\left(\overline{I V}_{\text {artery }}\right)$ subtracted by the mean intensity value in the adjacent tissue $\left(\overline{I V}_{\text {tissue }}\right)$ is considered the contrast between the artery and the tissue. The noise is defined as the standard deviation of the intensity of the adjacent tissue $\left(S D_{\text {tissue }}\right)$. These values were assessed by one observer (SJ), by drawing a region of interest (ROI) in patent arteries of femoropopliteal, crural, and foot angiographies, and a ROI in adjacent tissue. The ROI in the artery was drawn distally from the contrast injection site. All CNRs were calculated using the DSA images.

The pain score of the patient during contrast injection on a 4-point scale (no, mild, moderate, or severe pain), and volume of contrast used was recorded by the interventional radiologist performing the procedure.

\section{Sample size}

In a previous study assessing physicians confidence scores, the mean confidence score for therapeutic decision making based on DSA in patients with PAD was $8.1 \pm 1.4$ (standard deviation, SD) on a scale of 10 , with 0 being absolutely uncertain and 10 being absolutely certain [12]. In our design, the radiologists were asked to rate confidence on a scale of 0 to $100 \%$. Therefore, the SD of the confidence score was 14 . Since using a lower iodine concentration would be beneficial for the patient, we set the boundary for acceptable loss of confidence in the 240 or 140 group compared to the control group at $10 \%$.

To evaluate whether the mean difference (delta) of maximal $10 \%$ of the 240 or 140 group is not inferior to the 300 group, with a power of $80 \%(\mathrm{Zb}=0.842)$ and significance level of $0.10(\mathrm{Za}=1.28)$, and under the assumption that the SD of the groups would be 14,18 patients were needed per group. Therefore, 60 patients were included in total.

\section{Randomization}

Randomization was computer-based in a 1:1:1 ratio using minimization (biased coin randomization). Stratification was applied for the Fontaine classification (stage II versus stage III and IV) and presence of diabetes with the coin bias factor set at $80 \%$. Randomization was performed by the coordinating investigator after informed consent was obtained.

\section{Blinding}

To ensure that the interventional radiologist was blinded for the contrast concentration, the coordinating investigator supplied the contrast directly for the power injector and for contrast injection by hand. Patients were also blinded to the allocated contrast concentration.

\section{Statistical analysis}

The confidence of the interventional radiologist in diagnosing or in treating arterial stenosis or occlusion of the 240 and 140 groups was compared separately to the control group by a one-sided Mann-Whitney U test.

The volume of contrast material used in the 240 and 140 groups per patient was compared separately to the control group by two-sided independent samples $t$-test.

The interobserver variation in confidence in diagnosing arterial stenosis or occlusion and in grading imaging quality was evaluated by means of weighted kappa statistics including $95 \%$ confidence interval $(95 \% \mathrm{CI})$.

The CNR scores were compared between groups using the Mann-Whitney U test.

The pain scores during contrast injection were compared between groups using the $X^{2}$ test.

All analyses were done using SPSS (SPSS 20 Inc., Chicago, IL, USA). A $p$-value $<0.05$ was used as an indicator for statistical significance.

\section{Results}

\section{Intervention}

Single above the knee interventions were performed in 42 (70\%) patients, single below the knee interventions in eight (13\%) patients, and both above and below the knee interventions in five $(8 \%)$ patients. In five $(8 \%)$ patients no intervention was performed. One patient was evaluated for dissection, which could not be treated endovascularly, in two patients an occlusion could not be passed with the catheter, and in two patients no significant stenosis was found (false positive duplex ultrasound). Out of 60 angiographies, 59 femoropopliteal, 53 crural, and 52 lateral foot angiographies 
Table 2 Intervention characteristics

\begin{tabular}{lccc}
\hline & $\begin{array}{l}300 \text { group } \\
\text { (control) } \\
\mathrm{n}(\%)\end{array}$ & $\begin{array}{l}\text { 240 group } \\
\mathrm{n}(\%)\end{array}$ & $\begin{array}{l}140 \text { group } \\
\mathrm{n}(\%)\end{array}$ \\
\hline Number of patients & 21 & 19 & 20 \\
ATK intervention & $14(67)$ & $13(68)$ & $15(75)$ \\
BTK intervention & $2(10)$ & $4(21)$ & $2(10)$ \\
ATK/BTK intervention & $3(14)$ & $0(0)$ & $2(10)$ \\
No intervention & $2(10)$ & $2(11)$ & $1(5)$ \\
Total interventions & 26 & 20 & 22 \\
PTA & $15(58)$ & $11(55)$ & $15(68)$ \\
PTA+stent & $6(23)$ & $4(20)$ & $1(5)$ \\
Subintimal angioplasty & $3(11)$ & $4(20)$ & $3(13.5)$ \\
Subintimal+stent & $2(8)$ & $1(5)$ & $3(13.5)$ \\
\hline
\end{tabular}

$A T K$, above the knee (i.e. femoropopliteal arterial segments); BTK, below the knee; PTA, percutaneous transluminal angioplasty

were available for evaluation by the three observers. One femoropopliteal angiography was not made since the superficial femoral artery was proximally occluded. Seven crural and eight lateral foot angiographies were not made according to the standard operating procedure, since the tip of the catheter could not be placed at the level knee joint in the popliteal artery due to proximal occlusions in five patients, or due to the risk of dissection during advancement of the catheter in the artery in one patient, or since the radiologist refrained from imaging due to pain complaints during contrast injection in two patients.

In total 68 lesions were treated, of which 66 (97\%) were technically successful. Revascularization was not successful in one patient in the control group and one in the 140 group. Intervention characteristics are listed in Table 2.

\section{Confidence scores}

The median confidence score of the radiologist in diagnosing arterial lesions was $100 \%$ (range $81-100 \%$ ) for the control group. The median confidence scores in the 240 and 140 groups were respectively $100 \%$ (range $82-100 \%$ ) and $100 \%$ (range 91-100\%). The confidence scores of the 240 $(p=1.00)$ and 140 group $(p=1.00)$ were not inferior compared to the control group.
The median confidence score of the radiologist for treating arterial lesions was $100 \%$ (range 78-100\%) for the control group. The median scores in the 240 and 140 groups were respectively $100 \%$ (range $79-100 \%$ ) and $100 \%$ (range 63 $100 \%$ ). Confidence scores for the 240 and 140 groups were not inferior compared to the control group, with $p$-values of respectively 0.40 and 0.25 (Table 3 ).

\section{Volume of contrast used}

In the control group the interventional radiologist used a mean of $99 \pm 21 \mathrm{~mL}$ of contrast per patient. For the 240 and 140 groups this was respectively $101 \pm 32 \mathrm{~mL}$ and $127 \pm 40 \mathrm{~mL}$, with $p$ values compared with control-group of respectively 0.77 and 0.008. For the control group this resulted in an iodine load per patient of $29.7 \pm 6.3 \mathrm{~g}$, and for the 240 and 140 groups respectively in a load of $24.3 \pm 7.6 \mathrm{~g}$ and $17.8 \pm 5.6 \mathrm{~g}$. The iodine loads in the 240 and 140 groups were both significantly lower than the control group, with respective $p$-values of 0.022 and $<0.001$. See Table 4 for the contrast volume and iodine load per group.

\section{Image quality}

In all patients, the allocated contrast concentration was used and was adequate for diagnostic evaluation and intervention in all patients according to the interventional radiologist performing the procedure. The radiologist performing the intervention scored one crural acquisition as limited quality in the control group, and one pedal acquisition as non-diagnostic and limited quality. One pedal acquisition in the 240 group was scored as limited quality. In the 140 group, no acquisition was scored as non-diagnostic or limited quality. For all other acquisitions, the radiologist performing the procedure scored the images as diagnostic or exemplary quality (Fig. 1).

For the two radiologists assessing all standardized image acquisitions, one radiologist scored a pedal acquisition as nondiagnostic, and two pedal acquisitions as limited quality in the control-group. In the 240 group, one radiologist scored one crural angiography and one pedal angiography as limited quality. Both radiologists scored in the 140 group one pedal angiography as limited quality. All other angiographies were either scored as diagnostic or exemplary by both observers.
Table 3 Confidence scores of radiologists

\begin{tabular}{lllc}
\hline & $\begin{array}{l}300 \text { group } \\
\text { (control) } \\
\mathrm{n}(\%)\end{array}$ & $\begin{array}{l}240 \text { group } \\
\mathrm{n}(\%)\end{array}$ & $\begin{array}{l}140 \text { group } \\
\mathrm{n}(\%)\end{array}$ \\
\hline $\begin{array}{l}\text { Confidence in diagnosing arterial lesion in \% (range) } \\
p \text {-value* }\end{array}$ & $\begin{array}{l}100(81-100) \\
\text { NA }\end{array}$ & $\begin{array}{c}100(82-100) \\
1.0\end{array}$ & $100(91-100)$ \\
$\begin{array}{l}\text { Confidence in treating arterial lesions in \% (range) } \\
p \text {-value* }\end{array}$ & $100(78-100)$ & $100(79-100)$ & $100(63-100)$ \\
& $\mathrm{NA}$ & 0.40 & 0.25 \\
\hline
\end{tabular}

${ }^{*} p$-values given are compared to the control group. 
Table 4 Secondary outcomes

\begin{tabular}{|c|c|c|c|c|c|}
\hline & $\begin{array}{l}300 \text { group } \\
\text { (control) } \\
\mathrm{n}(\%)\end{array}$ & $\begin{array}{l}240 \text { group } \\
n(\%)\end{array}$ & $\begin{array}{l}p \text {-value } 240 \\
\text { vs. control }\end{array}$ & $\begin{array}{l}140 \text { group } \\
\text { n }(\%)\end{array}$ & $\begin{array}{l}p \text {-value } 140 \\
\text { vs. control }\end{array}$ \\
\hline Volume contrast used in $\mathrm{ml}(\mathrm{SD})^{\mathrm{T}}$ & $99(21)$ & $101(32)$ & 0.77 & $127(40)$ & 0.008 \\
\hline Iodine load in grams $(\mathrm{SD})^{\mathrm{T}}$ & $29.7(6.3)$ & $24.3(7.6)$ & 0.022 & $17.8(5.6)$ & $<0.001$ \\
\hline \multicolumn{6}{|l|}{$\mathrm{CNR}$ in median $(\mathrm{IQR})^{\mathrm{M}}$} \\
\hline Total & $34.9(23.0-44.0)$ & $27.2(17.9-46.6)$ & 0.13 & $28.0(17.5-38.7)$ & 0.083 \\
\hline Femoropopliteal & $35.0(28.3-44.2)$ & $44.4(27.2-50.7)$ & 0.43 & $34.8(20.9-62.5)$ & 0.68 \\
\hline Crural & $33.9(22.9-53.8)$ & $28.9(20.5-61.2)$ & 0.74 & $25.1(12.7-37.9)$ & 0.082 \\
\hline Foot & $30.8(13.2-38.9)$ & $13.9(11.6-19.6)$ & 0.018 & $25.5(14.2-35.9)$ & 0.74 \\
\hline Pain during contrast injection* & 300 group & 240 group & 140 group & & \\
\hline No & 6 & 11 & 17 & & \\
\hline Mild & 3 & 2 & 2 & & \\
\hline Moderate & 3 & 5 & 0 & & \\
\hline Severe & 8 & 1 & 0 & & \\
\hline$p$-value compared to control ${ }^{\mathrm{x}}$ & NA & 0.055 & 0.001 & & \\
\hline
\end{tabular}

$\mathrm{CNR}$, contrast-to-noise ratio; IQR, interquartile range; $\mathrm{SD}$, standard deviation

* In two patients, severity of pain was not assessed, since one patient was sedated. For the other patient pain was not recorded

NA, not assessed

${ }^{\mathrm{T}}$ Compared using two-sided independent samples $t$ test

M Compared using Mann-Whitney U test

${ }^{\mathrm{X}}$ Compared using $X^{2}$ test

\section{Arterial stenosis or occlusion}

A total of 408 out of 480 possible arterial segments were assessed for patency. As mentioned, due to occlusions, risk of dissection or pain complaints, 38 arteries could not be imaged according to protocol. Furthermore, either the plantar artery or dorsal pedal artery was not visualized due to proximal occlusion in 33 patients and were, therefore, scored as
Fig. 1 Standardized angiographic acquisitions using (A) 300, (B) 240, and (C) $140 \mathrm{mgI} / \mathrm{mL}$
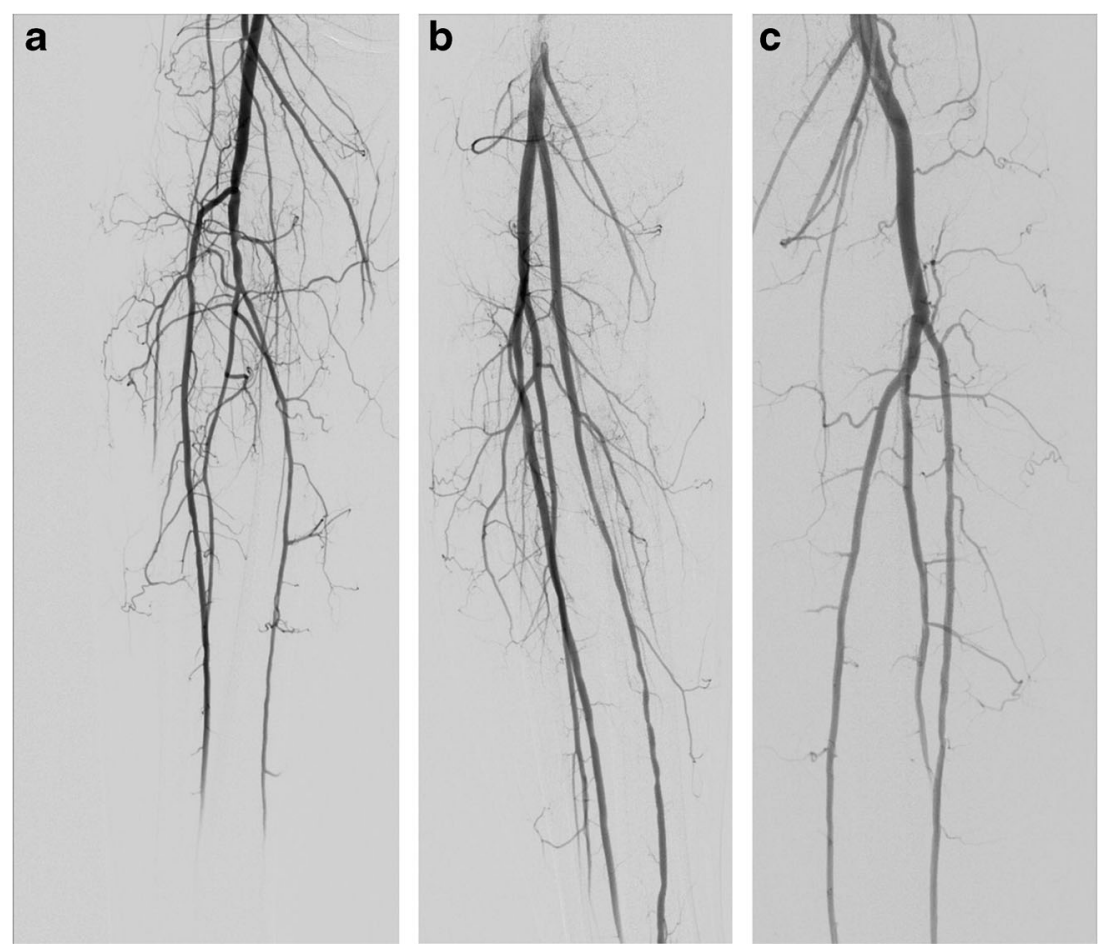
"not assessable". In one patient, the tibioperoneal trunk was absent due to anatomical variation. Interobserver agreement was highest for the control-group with a weighted kappa value of 0.85 (95\% $\%$, 0.77-0.93). Interobserver agreement was also excellent for the 240 and 140 groups with weighted kappa values of respectively $0.82(95 \% \mathrm{CI}, 0.72-0.91)$ and 0.81 (95\%CI, 0.72-0.91). See Table 5 for the interobserver agreement of arterial lesions between both radiologists.

\section{Contrast-to-noise ratio}

The CNR was highest for the control-group with a median ratio of 34.9 (IQR 23.0-44.0) . The CNR for the 240 and 140 groups was 27.2 (IQR 17.9-46.6) and 28.0 (IQR 17.5-38.7), with respective $p$-values of 0.13 and 0.083 compared to the control group. In general, the CNR was highest in the femoropopliteal arteries and lowest in the pedal arteries. See Table 4 for the CNR scores.

Table 5 Interobserver agreement of arterial lesions per group

\begin{tabular}{lcccc}
\hline \multicolumn{5}{c}{ CONTROL GROUP } \\
\multicolumn{5}{c}{ Radiologist 2} \\
Radiologist 1 & Patent & Stenosis $>50 \%$ & Occlusion & Total \\
Patent & 85 & 1 & 2 & 88 \\
Stenosis $>50 \%$ & 3 & 20 & 0 & 23 \\
Occlusion & 2 & 3 & 24 & 29 \\
Total & 90 & 24 & 26 & 140
\end{tabular}

20 arteries were not assessable.

Kappa $=0.85$ (95 \%CI 0.77-0.93)

240 GROUP

\begin{tabular}{lcccc}
\multicolumn{5}{l}{ Radiologist 2} \\
Radiologist 1 & Patent & Stenosis $>50 \%$ & Occlusion & Total \\
Patent & 75 & 2 & 2 & 79 \\
Stenosis $>50 \%$ & 6 & 14 & 2 & 22 \\
Occlusion & 0 & 1 & 28 & 29 \\
Total & 81 & 17 & 32 & 130
\end{tabular}

30 arteries were not assessable

Kappa $=0.82(95 \%$ CI 0.72-0.91)

140 GROUP

\begin{tabular}{lcccc}
\multicolumn{5}{l}{ Radiologist 2} \\
Radiologist 1 & Patent & Stenosis $>50 \%$ & Occlusion & Total \\
Patent & 80 & 1 & 3 & 84 \\
Stenosis $>50 \%$ & 6 & 20 & 1 & 27 \\
Occlusion & 1 & 2 & 24 & 27 \\
Total & 87 & 23 & 28 & 138
\end{tabular}

22 arteries were not assessable

Kappa $=0.81(95 \%$ CI 0.72-0.91)

Interobserver agreement in scoring standardized angiographies for the presence of arterial stenosis more than $50 \%$ and occlusion. Radiologist 1, DDB (3 years experience in interventional radiology); Radiologist 2 , $\mathrm{SS} n 1$ year experience in interventional radiology)

\section{Pain during contrast injection}

During contrast injection $14(70 \%)$ patients in the control group had some pain, which was severe in eight (40\%). Seven (42\%) patients in the 240 group had some pain during contrast injection. In the 140 group, 17 (89\%) patients had no pain and two $(11 \%)$ patients had mild pain during contrast injection. The $X^{2}$ test showed no significant difference in pain scores between the control group and the 240 group ( $p=$ 0.055 ) and a significant difference between the control group and the 140 group $(p=0.001)$. See Table 4 for the pain scores.

\section{Discussion}

This trial showed that the confidence of the interventional radiologist in diagnosing and treating lower limb arterial stenoses or occlusions in patients with PAD in the 240 and 140 groups were not inferior to the control group. The volume of contrast used in the 140 group was larger than in the control group and the 240 group. However, the iodine load in the 140 group was significantly lower, with a reduction of $40 \%$ compared to the standard contrast concentration. The image quality for the femoropopliteal, crural, and foot angiographies was generally diagnostic or exemplary. Moreover, the CNR remained relatively high enough for adequate diagnosis and treatment. Furthermore, patients in the 140 group had significantly less discomfort during contrast injection.

\section{Implications for clinical practice}

As recommended by the updated European Society of Urogenital Radiology (ESUR) Contrast Media Safety Committee guidelines, unnecessarily large contrast doses should be avoided to prevent CIN, and low or iso-osmolar contrast media should be used [1]. Furthermore, it has been shown that patients receiving an iodine load (in grams) higher than their estimated glomerular filtration rate eGFR value (in $\mathrm{mL} / \mathrm{min}$ ) are significantly more likely to develop CIN [13], and that in patients with occlusive vascular disease, a higher cumulative contrast agent exposure was an independent predictor of renal failure and death [14]. Since the iodine load is significantly reduced with lower concentrations, and confidence during intervention and image quality remained high, future lower limb arterial interventions, i.e. distal from the common femoral artery, in patients with PAD should be performed with the $140 \mathrm{mgI} / \mathrm{mL}$ contrast. As this study did not evaluate the incidence of CIN between groups, future studies should evaluate the effect of using lower contrast concentrations on the incidence of CIN.

Substitution of iodinated contrast media by carbon dioxide or gadolinium-based contrast media has been studied extensively. However, for carbon dioxide, studies reported poor 
visualization of the infrapopliteal arteries and discomfort in patients [15-21]. Likewise, gadolinium chelates are expensive and can only be used in low concentrations, resulting in low attenuation and image quality, especially in distal vessels, compared to iodinated contrast material $[22,23]$. Moreover, gadolinium does more harm to renal function than equal concentrations of iodinated contrast medium [24, 25].

\section{Limitations}

Patients with renal insufficiency, i.e. serum creatinine equal to or higher than $130 \mu \mathrm{mol} / \mathrm{L}$, were excluded in this trial, since we anticipated that participating in this trial could result in a higher contrast load compared to current clinical practice. However, in hindsight, the iodine load for the patients participating in this trial on average was as low as $24.0 \mathrm{mgI}$, and especially in the 240 and 140 groups. Since patients with renal insufficiency have a higher risk for CIN [3] and we excluded these patients, a relevant patient population was missed. Yet in our opinion, the results of this trial are also applicable for this patient population, since we do not expect a correlation between the outcomes of this trial and renal function.

The initial design of this study included the assessment of serum creatinine within 3 days following intervention to assess whether CIN had occurred to have direct evidence of the effect of using lower iodine concentrations on renal function. However, early in the trial this was removed from protocol, since patients were reluctant to reappear at the medical centre for blood withdrawal. We judged that we could refrain from creatinine assessment without compromising the study question, since the sample size calculations of this trial were based on confidence scores and not on the incidence of CIN. To evaluate whether there would be a difference between groups in the incidence of CIN, would have required a considerably larger study population.

Finally, our study showed that using a lower concentration leads to a higher injected volume of contrast. One could argue, therefore, that this could lead to an increased risk of CIN, since studies already have shown that a larger volume of contrast increases the risk of CIN $[9,26,27]$. However, as the number of milligrams iodine injected is a derivative of the volume injected, our opinion is that it is not the volume injected which increases the risk of CIN, but merely the milligrams of iodine injected.

\section{Conclusion}

Using an iodine contrast concentration of $140 \mathrm{mgI} / \mathrm{mL}$ will lead to a significant reduction of administered iodine load, thereby minimizing the risk for CIN, without compromising image quality. Use of low iodine dose contrast during DSA in PAD patients is recommended.
Acknowledgments This randomized controlled trial has been presented at CIRSE in September 2015. The scientific guarantor of this publication is Prof. Dr. J.A. Reekers. The authors of this manuscript declare no relationships with any companies, whose products or services may be related to the subject matter of the article. This study has received funding by GE Healthcare. GE had no influence in analysing and reporting of this trial. No complex statistical methods were necessary for this paper. Institutional Review Board approval was obtained. Written informed consent was obtained from all subjects (patients) in this study. Some study subjects or cohorts have not been previously reported.

Methodology: prospective, randomised controlled trial, multicenter study.

Open Access This article is distributed under the terms of the Creative Commons Attribution-NonCommercial 4.0 International License (http:// creativecommons.org/licenses/by-nc/4.0/), which permits any noncommercial use, distribution, and reproduction in any medium, provided you give appropriate credit to the original author(s) and the source, provide a link to the Creative Commons license, and indicate if changes were made.

\section{References}

1. Stacul F, van der Molen AJ, Reimer P et al (2011) Contrast induced nephropathy: updated ESUR Contrast Media Safety Committee guidelines. Eur Radiol 21:2527-2541

2. Morcos SK, Thomsen HS, Webb JA (1999) Contrast-media-induced nephrotoxicity: a consensus report. Eur Radiol 9:1602-1613

3. Morcos SK (2005) Prevention of contrast media-induced nephrotoxicity after angiographic procedures. J Vasc Interv Radiol 16:1323

4. Golshahi J, Nasri H, Gharipour M (2014) Contrast-induced nephropathy; A literature review. J Nephropathol 3:51-56

5. Ukaigwe A, Karmacharya P, Mahmood M et al (2014) Metaanalysis on efficacy of statins for prevention of contrast-induced acute kidney injury in patients undergoing coronary angiography. Am J Cardiol 114:1295-1302

6. Seyferth W, Dilbat G, Zeitler E (1983) Efficacy and safety of digital subtraction angiography with special reference to contrast agents. Cardiovasc Intervent Radiol 6:265-270

7. Naisby GP, Owen JP, Alexander TW, Cope L, Laker MF, Hamilton PJ (1991) Transfemoral digital subtraction aortography. Are diluted high osmolar contrast media acceptable? Acta Radiol 32:137-140

8. Ugolotti U, Larini P, Marcato C, Cusmano F, Puccianti F (1994) Peripheral arteriography with a new nonionic agent: comparison of iomeprol with iopamidol. Eur J Radiol 18:S77-S82

9. Ohno I, Hayashi H, Aonuma K et al (2013) Guidelines on the use of iodinated contrast media in patients with kidney disease 2012: digest version : JSN, JRS, and JCS Joint Working Group. Clin Exp Nephrol 17:441-479

10. Moher D, Schulz KF, Altman DG (2001) The CONSORT statement: revised recommendations for improving the quality of reports of parallel-group randomised trials. Lancet 357:1191-1194

11. Radiological Society of North America Radlex Web site. Image quality. Available via http://www.radlex.org/RID/RID10. Accessed 20 Nov 2014

12. Adriaensen ME, Kock MC, Stijnen T et al (2004) Peripheral arterial disease: therapeutic confidence of $\mathrm{CT}$ versus digital subtraction angiography and effects on additional imaging recommendations. Radiology 233:385-391

13. Nyman U, Björk J, Aspelin P, Marenzi G (2008) Contrast medium dose-to-GFR ratio: a measure of systemic exposure to predict 
contrast-induced nephropathy after percutaneous coronary intervention. Acta Radiol 49:658-667

14. Kougias P, Sharath S, Barshes NR et al (2014) Impact of cumulative intravascular contrast exposure on renal function in patients with occlusive and aneurysmal vascular disease. J Vasc Surg 59:1644 1650

15. Bees NR, Beese RC, Belli AM, Buckenham TM (1999) Carbon dioxide angiography of the lower limbs: initial experience with an automated carbon dioxide injector. Clin Radiol 54:833-838

16. Díaz LP, Pabón IP, García JA, de la Cal López MA (2000) Assessment of $\mathrm{CO} 2$ arteriography in arterial occlusive disease of the lower extremities. J Vasc Interv Radiol 11:163-169

17. Madhusudhan KS, Sharma S, Srivastava DN et al (2009) Comparison of intra-arterial digital subtraction angiography using carbon dioxide by 'home made' delivery system and conventional iodinated contrast media in the evaluation of peripheral arterial occlusive disease of the lower limbs. J Med Imaging Radiat Oncol 53:40-49

18. Ho CF, Chern MS, Wu MH et al (2003) Carbon dioxide angiography in lower limbs: a prospective comparative study with selective iodinated contrast angiography. Kaohsiung J Med Sci 19:599-607

19. Oliva VL, Denbow N, Thérasse E et al (1999) Digital subtraction angiography of the abdominal aorta and lower extremities: carbon dioxide versus iodinated contrast material. J Vasc Interv Radiol 10: 723-731

20. Rolland Y, Duvauferrier R, Lucas A et al (1998) Lower limb angiography: a prospective study comparing carbon dioxide with iodinated contrast material in 30 patients. Am J Roentgenol 171: 333-337

21. Seeger JM, Self S, Harward TR, Flynn TC, Hawkins IF Jr (1993) Carbon dioxide gas as an arterial contrast agent. Ann Surg 217: 688-697

22. Hammer FD, Goffette PP, Malaise J, Mathurin P (1999) Gadolinium dimeglumine: an alternative contrast agent for digital subtraction angiography. Eur Radiol 9:128-136

23. Wagner HJ, Kalinowski M, Klose KJ, Alfke H (2001) The use of gadolinium chelates for X-ray digital subtraction angiography. Invest Radiol 36:257-265

24. Nyman U, Elmståhl B, Leander P, Nilsson M, Golman K, Almén T (2002) Are gadolinium-based contrast media really safer than iodinated media for digital subtraction angiography in patients with azotemia? Radiology 223:311-318

25. Erley CM, Bader BD, Berger ED et al (2004) Gadolinium-based contrast media compared with iodinated media for digital subtraction angiography in azotaemic patients. Nephrol Dial Transplant 19: 2526-2531

26. Dangas G, Iakovou I, Nikolsky E et al (2005) Contrast-induced nephropathy after percutaneous coronary interventions in relation to chronic kidney disease and hemodynamic variables. Am J Cardiol 95:13-19

27. Yamamoto M, Hayashida K, Mouillet G et al (2013) Renal function-based contrast dosing predicts acute kidney injury following transcatheter aortic valve implantation. JACC Cardiovasc Interv 6:479-486 